\title{
ADESÃO DE IDOSOS COM DIABETES MELLITUS À TERAPÊUTICA: REVISÃO INTEGRATIVA*
}

\author{
Sheila Silveira Costa ${ }^{1}$, Rita Arim Rosales ${ }^{2}$, Janaína Amorim de Ávila ${ }^{3}$, Marlene Teda Pelzer ${ }^{4}$, Celmira Lange ${ }^{5}$
}

\begin{abstract}
RESUMO: Objetivou-se identificar na literatura os fatores que influenciam a adesão do idoso com Diabetes Mellitus à terapêutica. Realizou-se revisão integrativa, com análise textual qualitativa. A busca ocorreu no Portal de Periódicos da Coordenação de Aperfeiçoamento de Pessoal de Nível Superior, por publicações brasileiras, em oito bases de dados, no período de janeiro de 2002 a setembro de 2016. Selecionou-se 12 estudos, dos 187 encontrados, originando duas categorias: Fatores que influenciam positivamente a adesão do idoso com Diabetes Mellitus à terapêutica, e Fatores que influenciam negativamente a adesão do idoso com Diabetes Mellitus à terapêutica. Conclui-se a necessidade de mais estudos sobre a temática, pois foram encontradas apenas publicações com baixos níveis de evidências e a premência da intensificação no diálogo conscientizador com os idosos e suas famílias, no intuito de maximizar e/ou manter sua qualidade de vida, autonomia e independência. DESCRITORES: Revisão; Idoso; Diabetes Mellitus; Terapêutica; Enfermagem.
\end{abstract}

\section{ADHERENCE TO THERAPY BY ELDERLY PATIENTS WITH DIABETES MELLITUS: AN INTEGRATIVE REVIEW}

ABSTRACT: The present study had the aim of identifying in the literature the factors that influence adherence to therapy by elderly individuals with diabetes mellitus. An integrative review with qualitative textual analysis was conducted. The search was conducted at the Portal of Periodicals of the Coordination for the Improvement of Higher Education Personnel and included Brazilian publications in eight databases between January 2002 and September 2016. A total of 12 studies was selected from the 187 that were found, generating two categories: factors that influence adherence to therapy by elderly individuals with diabetes mellitus positively; and factors that influence adherence to therapy by elderly individuals with diabetes mellitus negatively. The fact that only publications with low levels of evidence were found revealed the need for further studies on the theme and intensification of the awarenessraising dialogue with elderly individuals and their families in order to maximize and/or maintain their quality of life, autonomy, and independence.

DESCRIPTORS: Review; Aged; Diabetes Mellitus; Therapeutics; Nursing.

\section{ADHESIÓN DE ANCIANOS CON DIABETES MELLITUS A LA TERAPÉUTICA: REVISIÓN INTEGRATIVA}

RESUMEN: Se objetivó identificar en la literatura los factores que influyen en adhesión del anciano con Diabetes Mellitus a la terapéutica. Se realizó revisión integrativa, con análisis textual cualitativo. Búsqueda efectuada en Portal de Periódicos de la Coordinación de Perfeccionamiento de Personal de Nivel Superior, por publicaciones brasileñas, en ocho bases de datos, de enero de 2002 a setiembre de 2016. Doce estudios fueron seleccionados de 187 hallados, determinando dos categorías: Factores que influyen positivamente en adhesión del anciano con Diabetes Mellitus a la terapéutica; y Factores que influyen negativamente en adhesión del anciano con Diabetes Mellitus a la terapéutica. Se concluye en que son necesarios más estudios sobre la temática, pues solo fueron encontradas publicaciones con bajos niveles de evidencias, y la urgencia de la identificación del diálogo de concientización con los ancianos y sus familias, para maximizar y/o mantener su calidad de vida, autonomía e independencia.

DESCRIPTORES: Revisión; Anciano; Diabetes Mellitus; Terapéutica; Enfermería.

*Artigo extraído da dissertação intitulada: "Gestão do regime terapêutico da pessoa idosa com diabetes mellitus tipo 2". Universidade Federal do Rio Grande, 2015.

${ }^{1}$ Enfermeira. Mestre em Enfermagem. Enfermeira no Hospital Universitário Dr. Miguel Riet Corrêa Jr. Rio Grande, RS, Brasil. ${ }^{2}$ Enfermeira. Doutoranda em Enfermagem na Universidade Federal do Rio Grande. Rio Grande, RS, Brasil.

${ }^{3}$ Enfermeira. Doutoranda em Enfermagem da Escola na Universidade Federal de Pelotas. Pelotas, RS, Brasil.

${ }^{4}$ Enfermeira. Doutora em Enfermagem. Docente de Enfermagem e do Programa de Pós-Graduação em Enfermagem da Universidade Federal do Rio Grande. Rio Grande, RS, Brasil.

${ }^{5}$ Enfermeira. Doutora em Enfermagem. Docente de Enfermagem e do Programa de Pós-Graduação em Enfermagem da Universidade Federal de Pelotas. Pelotas, RS, Brasil. 


\section{INTRODUÇÃO}

O Diabetes Mellitus (DM) acomete mais de 382 milhões de pessoas no mundo, destes, 11,9 milhões são brasileiros ${ }^{(1)}$. As cidades com índices mais elevados no Brasil são Fortaleza/CE - 7,3\%; Vitória/ES 7,1\%; e Porto Alegre/RS - 6,3\% ${ }^{(2)}$. Destaca-se, ainda, o aumento do DM em pessoas com mais de 65 anos de idade, configurando-se como uma das Doenças Crônicas Não Transmissíveis (DCNT) mais comuns em idosos ${ }^{(3)}$.

O DM decorre de distúrbios no metabolismo da glicose, das gorduras e das proteínas, resultando em hiperglicemia. Desencadeia impactos de ordem emocional/psicológica, financeira e social, além de complicações que podem causar disfunções e/ou insuficiências oculares, renais, neurológicas, cardíacas e vasculares ${ }^{(2)}$.

Os comprometimentos oriundos do DM, associados ao processo de envelhecimento, afetam sobremaneira a qualidade de vida, autonomia e independência da pessoa idosa. No intuito de evitar os agravos dessa moléstia, necessita-se modificar comportamentos prejudiciais no cotidiano, rever os hábitos alimentares, praticar atividades físicas, consultar periodicamente profissionais de saúde e utilizar medicamentos com prescrição médica. Para isso, fazem-se necessárias a aceitação e a conscientização sobre a doença, enfrentamento efetivo a fim de que ocorra a manutenção da qualidade de vida, promoção de saúde e a prevenção de subsequentes agravos.

Neste contexto, qualidade de vida pode ser entendida como a satisfação nos âmbitos da vida familiar, amorosa, social, ambiental e da própria estética existencial, abrangendo elementos como o conforto e bem-estar, entre outros, e comporta ainda uma série de significados que incluem conhecimentos, experiências e valores individuais e coletivos $^{(4)}$.

Na promoção da saúde e prevenção dos agravos, salienta-se que os enfermeiros são os profissionais que dispensam maior período de tempo de sua jornada de trabalho no acompanhamento às pessoas acometidas por DCNT. Diante disso, precisam atentar às reais necessidades das pessoas com diabetes, suas vivências e experiências, considerando o contexto social e individual de cada indivíduo, seu estilo de vida, aspectos culturais, emocionais, psicológicos e econômicos.

É por meio das intervenções - diagnósticos, tratamentos e acompanhamentos - que os profissionais exercem influência sobre os comprometimentos que podem gerar dependências e desconfortos. E, desta forma, contribuem para a diminuição dos danos e consequências inferidas pela doença ${ }^{(4)}$.

É imprescindível observar as possíveis consequências de uma adesão inadequada ou de não adesão ao tratamento, no intuito de amenizar o ônus financeiro com tratamentos avançados e melhorar a qualidade de vida das pessoas idosas. Compreende-se que diversos fatores podem influenciar esta decisão.

Este estudo se justifica pelos altos índices de morbimortalidade decorrentes do DM em pessoas idosas, pela diminuição da qualidade de vida, autonomia e independência, assim como pelos altos custos dos tratamentos advindos das complicações da doença. Este contexto pode ser amenizado/ revertido por meio de ações educativas em saúde, baseadas na identificação precoce dos fatores que influenciam a pessoa idosa em aderir ou não à proposta terapêutica. Dessa forma, espera-se que este estudo venha contribuir com os profissionais de saúde, facilitando a identificação de fatores que influenciam a adesão dos idosos à terapêutica em suas atividades práticas, e assim, favorecendo a intervenção precoce e eficaz.

Diante do exposto, este estudo tem como objetivo identificar na literatura os fatores que influenciam a adesão do idoso com Diabetes Mellitus à terapêutica.

\section{MÉTODO}

O estudo consistiu de uma revisão integrativa e seguiu as seguintes etapas: 1) Elaboração da questão de pesquisa; 2) Estabelecimento de critérios para inclusão e exclusão de estudos e busca na literatura; 3) Definição das informações a serem extraídas dos estudos e coleta de dados, cujo objetivo foi extrair 
dos artigos selecionados as informações relevantes, organizando-as; 4) Avaliação dos estudos incluídos na revisão integrativa; 5) Interpretação dos resultados; 6) Apresentação da revisão/síntese ${ }^{(5)}$.

Para definição da questão de pesquisa, utilizou-se a estratégia PICO, composta de quatro elementos indispensáveis para que a pergunta de pesquisa, suporte à busca bibliográfica: the patient, population, or problem (P); the intervention or independent variable (I); the comparison (C); and the dependent variables or outcome(s) of interest $(O)^{(6)}$. Dessa forma, teve-se como questão norteadora: Quais são os fatores identificados na literatura que influenciam a adesão do idoso portador de Diabetes Mellitus à terapêutica recomendada?

A busca transcorreu no Portal de Periódicos da Coordenação de Aperfeiçoamento de Pessoal de Nível Superior (CAPES), no mês de outubro de 2016. Foram utilizados os Descritores em Ciências da Saúde (DeCS), em português: "Idoso" e "Diabetes Mellitus", agrupados pelo operador boleano "AND", em oito bases de dados, tendo em vista os artigos divulgados no período de janeiro de 2002 a setembro de 2016.

Optou-se por estudos realizados em território brasileiro por exporem especificamente as condições de saúde do idoso no Brasil e por este espaço temporal para compreender o ano anterior e todo o período posterior à promulgação da Lei n. 10.741 de $1^{\circ}$ de outubro de $2003^{(7)}$, destinada a regular os direitos assegurados às pessoas idosas.

Na busca nas bases de dados, encontrou-se 54 estudos na Directory of Open Access Journals, 41 artigos na Scientific Electronic Library Online - SciELO Brazil, 37 na SciELO (CrossRef), 32 na Scopus (Elsevier), nove na Medical Literature Analysis and Retrieval System Online (Medline), seis na Science Citation Index Expanded, quatro na Social Sciences Citation Index e quatro na OneFile, totalizando 187 artigos. Destes, excluiu-se 128 estudos repetidos em mais de uma base de dados, 45 após leitura dos títulos, resumos e descritores, por não estarem em conformidade com a temática e/ou não contemplarem os critérios de inclusão e exclusão e dois após leitura na íntegra, permanecendo 12 artigos pertinentes à pesquisa.

Como critérios de inclusão dos estudos, optou-se por artigos disponíveis online na íntegra, nos idiomas português, inglês e espanhol, em bases de dados nacionais e internacionais e que abordassem a temática em estudo. Foram excluídas as publicações sem abordagem à adesão do idoso à terapêutica do Diabetes Mellitus, realizadas fora do território nacional, e com idiomas e períodos que não os estipulados nos critérios de inclusão.

Para possibilitar a avaliação e a interpretação dos dados, procedeu-se a seleção/categorização dos estudos por meio de um protocolo de análise e síntese de informações, que contemplou autoria, formação acadêmica dos autores, título do artigo, periódico, ano, resultados e nível de evidência.

Para efetuar a classificação dos estudos, optou-se pelo Sistema de Avaliação para Hierarquia de Evidências definidos por Melnyk e Fineout-Overholt ${ }^{(8)}$, inserido na medicina baseada em evidências, $\mathrm{o}$ qual classifica as evidências segundo o delineamento de pesquisa, ou seja, a abordagem metodológica, e inclui pesquisas quantitativas e qualitativas.

Assim, classifica-se como: nível 1, evidências oriundas de revisão sistemática ou metanálise de relevantes ensaios clínicos randomizados controlados ou oriundas de diretrizes clínicas baseadas em revisões sistemáticas de ensaios clínicos randomizados controlados; nível 2, evidências derivadas de pelo menos um ensaio clínico randomizado controlado bem delineado; nível 3, evidências obtidas de ensaios clínicos bem delineados sem randomização; nível 4, evidências provenientes de estudos de coorte e de caso-controle bem delineados; nível 5, evidências originárias de revisão sistemática de estudos descritivos e qualitativos; nível 6, evidências derivadas de um único estudo descritivo ou qualitativo; nível 7, evidências oriundas de opinião de autoridades e/ou relatório de comitês de especialistas $^{(8)}$.

A análise foi textual, método que possibilita identificar e isolar enunciados dos conteúdos a ele submetidos, categorizando esses enunciados e produzindo textos, de maneira a integrar descrição e interpretação ${ }^{(9)}$. As sínteses das informações foram apresentadas de forma analítica ${ }^{(5)}$. 
Os dados obtidos nos estudos selecionados são apresentados nos Quadros 1 e 2, constituídos por: Quadro 1 - Identificação, Título, Autoria/Ano/Área profissional, Periódico/Tipo de estudo; Quadro 2 Identificação e Resultados.

Quadro 1 - Apresentação das publicações selecionadas conforme o Título, Autoria/Ano/Área profissional, Periódico/Tipo de estudo. Rio Grande, RS, Brasil, 2016

\begin{tabular}{|c|c|c|c|}
\hline Estudo & Título & Autoria/Ano/Área profissional & $\begin{array}{l}\text { Periódico/Tipo de } \\
\text { estudo }\end{array}$ \\
\hline E1 & $\begin{array}{l}\text { Educação conscientizadora do } \\
\text { idoso diabético: uma proposta de } \\
\text { intervenção do Enfermeiro }\end{array}$ & $\begin{array}{l}\text { Tavares DMS, Rodrigues RAP }{ }^{(10)} \text {. } \\
2002 \text {. } \\
\text { Enfermagem }\end{array}$ & $\begin{array}{l}\text { Rev. esc. enferm. USP. } \\
\text { Descritivo / quanti - } \\
\text { qualitativo }\end{array}$ \\
\hline E2 & $\begin{array}{l}\text { Estudo de sobrevivência de uma } \\
\text { coorte de pessoas de } 60 \text { anos e mais } \\
\text { no município de Botucatu (SP) - Brasil }\end{array}$ & $\begin{array}{l}\text { Ruiz T, Chalita LVAS, Barros } \\
\text { MBA }^{(11)} .2003 . \\
\text { Médica e Estatística }\end{array}$ & $\begin{array}{l}\text { Rev. bras. epidemiol. } \\
\text { Inq. Populac. }\end{array}$ \\
\hline E3 & $\begin{array}{l}\text { Utilização medicamentosa por idosos } \\
\text { de uma Unidade Básica de Saúde da } \\
\text { Família de Fortaleza - CE }\end{array}$ & $\begin{array}{l}\text { Vasconcelos FF, Victor JF, } \\
\text { Moreira TMM, de Araújo TL }{ }^{(12)} \text {. } \\
2005 . \\
\text { Enfermagem }\end{array}$ & $\begin{array}{l}\text { Acta paul.enferm. } \\
\text { Descritivo / Transversal }\end{array}$ \\
\hline E4 & $\begin{array}{l}\text { Fatores associados à prevalência de } \\
\text { diabetes auto referido entre idosos de } \\
\text { Minas Gerais, } 2003\end{array}$ & $\begin{array}{l}\text { Viegas-Pereira APF, Rodrigues } \\
\text { RN, Machado CJ(13). } 2008 . \\
\text { Ciências Econômicas }\end{array}$ & $\begin{array}{l}\text { Rev. bras. estud. popul. } \\
\text { Estudo seccional }\end{array}$ \\
\hline E5 & $\begin{array}{l}\text { Fatores associados ao equilíbrio } \\
\text { funcional e à mobilidade em idosos } \\
\text { diabéticos ambulatoriais }\end{array}$ & $\begin{array}{l}\text { Cordeiro RC, Jardim JR, Perracini } \\
\text { MR, Ramos LR }{ }^{(14)} .2009 . \\
\text { Fisioterapia, Médica e Eng. } \\
\text { Eletrônica }\end{array}$ & $\begin{array}{l}\text { Arq Bras Endocrinol } \\
\text { Metab. } \\
\text { Expl./Transv. }\end{array}$ \\
\hline E6 & $\begin{array}{l}\text { Diabetes autor referido em idosos: } \\
\text { prevalência, fatores associados e } \\
\text { práticas de controle }\end{array}$ & $\begin{array}{l}\text { Francisco PMSB, Belon AP, } \\
\text { Barros MBA, Carandina L, Alves } \\
\text { MCGP, Goldbaum M, et al }{ }^{(15)} \text {. } \\
2010 . \\
\text { Estatística, Ciênc. Soc. e Médica }\end{array}$ & $\begin{array}{l}\text { Cad. Saúde Pública. } \\
\text { Transversal de base } \\
\text { populacional }\end{array}$ \\
\hline E7 & $\begin{array}{l}\text { Compreendendo o significado de } \\
\text { qualidade de vida segundo idosos } \\
\text { portadores de Diabetes Mellitus tipo } \\
\text { II }\end{array}$ & $\begin{array}{l}\text { Ribeiro JP, Rocha AS, Popim } \\
\text { RC }{ }^{(16)} \text {. } 2010 . \\
\text { Enfermagem }\end{array}$ & $\begin{array}{l}\text { Esc. Anna Nery. } \\
\text { Qualitativo }\end{array}$ \\
\hline E8 & $\begin{array}{l}\text { Diabetes mellitus: fatores associados } \\
\text { à prevalência em idosos, medidas } \\
\text { e práticas de controle e uso dos } \\
\text { serviços de saúde em São Paulo, Brasil }\end{array}$ & $\begin{array}{l}\text { Mendes TAB, Goldbaum M, } \\
\text { Segri NJ, Barros MBA,Cesar CLG, } \\
\text { AlvesMCGP(17).2011. } \\
\text { Médica e Estatística }\end{array}$ & $\begin{array}{l}\text { Cad. Saúde Pública. } \\
\text { Transversal de base } \\
\text { populacional }\end{array}$ \\
\hline E9 & $\begin{array}{l}\text { Efeito de um programa de educação } \\
\text { no nível de conhecimento e nas } \\
\text { atitudes sobre o diabetes mellitus }\end{array}$ & $\begin{array}{l}\text { Gandra FPP, Silva KC, Castro CF, } \\
\text { Esteves EA, Nobre LN }{ }^{(18)} .2011 . \\
\text { Nutrição }\end{array}$ & $\begin{array}{l}\text { Rev Bras Promoç Saúde. } \\
\text { Intervenção }\end{array}$ \\
\hline E10 & $\begin{array}{l}\text { Visão do mundo, cuidado cultural } \\
\text { e conceito ambiental: o cuidado do } \\
\text { idoso com Diabetes Mellitus }\end{array}$ & $\begin{array}{l}\text { Souza NMG, Honorato SMA, } \\
\text { Xavier ATF, Pereira FGF, de } \\
\text { Ataíde MBC }{ }^{(19)} .2012 . \\
\text { Enfermagem }\end{array}$ & $\begin{array}{l}\text { Rev. Gaúcha Enferm. } \\
\text { Desc./Qualit. }\end{array}$ \\
\hline E11 & $\begin{array}{l}\text { Avaliação da competência de idosos } \\
\text { diabéticos para o autocuidado }\end{array}$ & $\begin{array}{l}\text { Marques MB, da Silva MJ, } \\
\text { Coutinho JFV, Lopes MVO }{ }^{(20)} \text {. } \\
\text { 2013. Enfermagem }\end{array}$ & $\begin{array}{l}\text { Rev. esc. enferm. USP. } \\
\text { Expl./Transv. }\end{array}$ \\
\hline E12 & $\begin{array}{l}\text { Correlação da qualidade de vida com } \\
\text { conhecimento e atitude de idosos } \\
\text { diabéticos. }\end{array}$ & $\begin{array}{l}\text { de Sousa MC, Dias FA, } \\
\text { Nascimento JS, Tavares DMS }{ }^{(21)} \text {. } \\
2016 . \\
\text { Enfermagem e Terap. Ocup. }\end{array}$ & $\begin{array}{l}\text { Invest. educ. enferm. } \\
\text { Quant./Transv. }\end{array}$ \\
\hline
\end{tabular}




\begin{tabular}{|c|c|}
\hline Estudo & Resultados \\
\hline E1 & A Associação dos Diabéticos teve como objetivo a educação em saúde de seus frequentadores. \\
\hline E2 & $\begin{array}{l}\text { O nível sócio econômico influencia os riscos de mortalidade. Os homens que deixaram de ser chefes } \\
\text { de suas famílias apresentaram mais riscos de mortalidade dos que continuaram. }\end{array}$ \\
\hline E3 & $\begin{array}{l}\text { Em relação ao uso de medicamentos, alguns idosos utilizavam subdosagem, desconheciam o motivo } \\
\text { de tomarem de 1-4 compr./dia, referiram efeitos colaterais. Outros não têm ajuda familiar para tomar } \\
\text { a medicação, moram sozinhos, não são alfabetizados. }\end{array}$ \\
\hline E4 & $\begin{array}{l}\text { A chance de ter diabetes foi maior para os idosos residentes na área urbana; mulheres; hipertensos; } \\
\text { com doenças cardíacas; pensionistas; sem ou com baixa instrução. }\end{array}$ \\
\hline E5 & $\begin{array}{l}\text { Os idosos diabéticos demonstraram limitações de mobilidade associadas à fragilidade funcional } \\
\text { ou deficiências. Relataram elevado número de co-morbidades crônicas. Demonstraram baixo } \\
\text { desempenho adaptativo. }\end{array}$ \\
\hline E6 & $\begin{array}{l}\text { Houve diferença entre diabéticos e não diabéticos quanto à autoavaliação da saúde, internação, } \\
\text { morbidade autorreferida nos últimos } 15 \text { dias, e relato das seguintes doenças: hipertensão, anemia, } \\
\text { doença renal e cardiovascular. }\end{array}$ \\
\hline E7 & A restrição alimentar foi o ponto de maior repercussão na qualidade de vida dos idosos diabéticos. \\
\hline E8 & $\begin{array}{l}\text { As maiores taxas de diabetes em idosos foram associados à má ou muito má autoavaliação de saúde, } \\
\text { nunca ter consumido álcool ou ter parado de beber, viuvez, uma ou mais hospitalizações nos últimos } \\
12 \text { meses. }\end{array}$ \\
\hline E9 & $\begin{array}{l}\text { Observou-se aumento do número de respostas corretas sobre os sintomas e complicações do } \\
\text { diabetes, no reconhecimento da importância da atividade física e do autoaplicação de insulina. }\end{array}$ \\
\hline E10 & $\begin{array}{l}\text { Os idosos com DM2 sentem sua vida sob fragilidade constante, em um mundo de restrições, } \\
\text { entretanto, buscam garantir sua saúde através do cultivo à fé com a religiosidade, do uso das plantas } \\
\text { medicinais e do apoio dos profissionais de saúde. }\end{array}$ \\
\hline E11 & $\begin{array}{l}\text { Apenas } 6 \% \text { dos idosos diabéticos mostraram-se competentes para o autocuidado, sendo influenciados } \\
\text { pela baixa escolaridade e a diminuição da acuidade visual. }\end{array}$ \\
\hline E12 & $\begin{array}{l}\text { Quanto maior o conhecimento e atitude, maiores foram os escores de Qualidade de Vida nos } \\
\text { domínios físicos, psicológico, relações sociais e meio ambiente; e facetas funcionamentos dos } \\
\text { sentidos, autonomia e intimidade. }\end{array}$ \\
\hline
\end{tabular}

De acordo com a análise das bases de dados, observou-se a predominância de estudos indexados nas bases Directory of Open Access Journals $(\mathrm{n}=11,91 \%)$, seguida SciELO Brazil oito (68\%), SciELO (CrossRef) oito (68\%) e Scopus (Elsevier) cinco (41\%), demonstrando o alto índice de publicações nacionais indexadas em bases internacionais.

Em relação ao ano de divulgação dos estudos, verificou-se a publicação de três artigos no período compreendido entre 2002 e 2006, seis estudos entre 2007 e 2011 e três entre 2012 e 2016.

Quanto à área de atuação dos profissionais autores, constatou-se que cinco (41\%) dos estudos foram escritos exclusivamente por enfermeiros, um $(8,3 \%)$ por nutricionistas e seis $(50 \%)$ foram desenvolvidos por meio da ação conjunta entre profissionais das demais áreas.

Conforme a análise dos resultados e dos níveis de evidência, constatou-se que 11 artigos apresentaram nível de evidência 6 (91\%) e um nível 2 (8\%), ou seja, houve o predomínio de artigos envolvendo estudos do tipo qualitativos, descritivos e transversais. Sendo encontrado apenas um estudo com nível de evidência elevado.

Para possibilitar a análise, interpretação e discussão das informações obtidas, dividiu-se os resultados em categorias: Fatores que influenciam positivamente a adesão do idoso portador de DM à terapêutica, e Fatores que influenciam negativamente a adesão do idoso portador de DM à terapêutica.

\section{- DISCUSSÃO}




\section{Fatores que influenciam positivamente a adesão do idoso com DM à terapêutica}

A análise dos resultados dos estudos demonstrou a importância do suporte familiar ao idoso com DM, pois a convivência com a família e suas influências tornam-se expressivas nas relações de cuidado, oferecendo suporte psicológico, social, financeiro. Uma das formas de apoio foram alterações na alimentação, para torná-la mais saudável, contribuindo para a adaptação do idoso (E7) ${ }^{(16)}$. A família, ao adaptar-se à nova dieta, com vistas a apoiá-lo, previne e/ou retarda o aparecimento da doença, gerando benefícios mútuos $(\mathrm{E} 1)^{(10)}$. O repensar uma abordagem alimentar saudável, pode auxiliá-los, encorajando-os ao tratamento e ajudando-os em suas necessidades, de forma não controladora (E7) ${ }^{(16)}$.

A família deve ser conhecedora do processo terapêutico, no intuito de favorecer sua implementação e o autocuidado da pessoa idosa ${ }^{(22)}$, pois sentimentos negativos permeiam aqueles que convivem com DM, explicitado em pesquisa por $60 \%$ dos portadores, podendo acarretar em depressão, decorrente da deficiência de apoio social formal e informal ${ }^{(23)}$.

A religiosidade/espiritualidade também foi percebida como condição que auxilia no confronto com a doença. O resgate da fé é utilizado pelos idosos na busca da cura e da força para combater a moléstia $(\mathrm{E} 10)^{(19)}$.

As contribuições dos grupos de apoio se apresentam como forma de compartilhamento de experiências, dos anseios, das necessidades, das negações, dentre outros. Os grupos promovem processo reflexivo sobre o viver com diabetes e buscam manter a autonomia dos idosos, conscientizando-os acerca do autocuidado. A convivência com o outro em situação similar favorece e facilita o entendimento que é possível viver bem com a doença ${ }^{(24)}$.

Os profissionais de saúde, em seu labor, são valorizados pelos idosos, na medida em que suas intervenções, abordagens e orientações sejam resolutivas. Estudo sobre o cotidiano de oito idosos com Diabete Mellitus tipo 2 retratou que a maioria destes adotaram as orientações que lhes foram recomendadas durante as consultas, principalmente no que se referiu à terapia medicamentosa. Em relação às terapias alternativas complementares, constatou o uso de chás, advindo de outros conhecimentos e crenças populares. Esta prática, denominada fitoterapia, é uma das estratégias encontradas para a preservação do bem-estar da pessoa idosa (E10) ${ }^{(19)}$.

As expectativas que os idosos portadores de DM possuem em relação aos profissionais de saúde não se limitam ao fornecimento das informações do que se deve ou não fazer, e sim, que ocupem espaço de orientação e diálogo, com o desenvolvimento de confiança mútua, o que Ihes auxiliaria a manter uma conduta de cuidado para um viver com qualidade ${ }^{(24)}$. A educação em saúde aproxima família, profissional e paciente, propiciando troca de saberes entre os sujeitos, no intuito de promover adesão ao regime terapêutico e a qualidade de vida ${ }^{(22)}$.

\section{Fatores que influenciam negativamente a adesão do idoso com DM à terapêutica}

A baixa renda foi identificada como um dos fatores mais preponderantes e prejudiciais à aderência ao tratamento. Nos estudos analisados (E2, E3, E8, E11), os idosos recebiam em média 2,4 salários mínimos, demonstrando a dependência da renda familiar para a sobrevivência, e dessa forma, possuindo razões financeiras como um dos motivos para não aderência ao tratamento ${ }^{(11,12,17,20)}$. Este fato leva os idosos a assumir atitude inadequada, ou seja, "economizarem" remédios, não seguindo a terapêutica prescrita $(\mathrm{E} 3)^{(12)}$.

As dificuldades financeiras prejudicam a aquisição de medicamentos para o controle glicêmico, bem como a compra de alimentos adequados para o consumo. Embora algumas medicações sejam fornecidas pelo Sistema Único de Saúde, nem sempre estão disponíveis (E7). Os gastos com a doença não se limitam às questões de medicamentos e alimentação, mas também às tecnologias para controle glicêmico, como as fitas de hemoglucotestes; insumos, seringas, agulhas; gastos com consultas médicas; dentre outros. Com isso, a família necessita comprometer parte de seu orçamento mensal com a compra desses materiais tecnológicos (E7) $)^{(16)}$.

Outra questão que influencia negativamente a adesão à terapêutica consiste em conhecimentos limitados frente à doença. Uma das investigações (E11) demonstrou que apenas 6\% dos entrevistados 
foram considerados competentes para a administração de seus medicamentos ${ }^{(20)}$. Em outra (E3), 76,9\% dos idosos ingeriam de 1 a 4 comprimidos por dia, mas $23 \%$ desconheciam o motivo do uso diário de medicação, ressaltando que não era relacionado à presença da hipertensão e/ou do diabetes mellitus, nem aos sintomas e complicações decorrentes dessas doenças ou os efeitos colaterais advindos dos medicamentos ${ }^{(12)}$. Foi observada (E2, E4, E8, E10, E12) uma menor prevalência de diabéticos entre os idosos com maior escolaridade. Dentre os idosos diabéticos, 20,6\% estudaram "8 anos ou mais", comparados a 30,5\% entre os não diabéticos ${ }^{(11,13,17,19,25)}$.

Corroborando, pesquisa comparou as condições de saúde dos idosos com DM, segundo as variáveis sexo e faixa etária, no município de Uberaba/MG, e evidenciou que 63,4\% dos idosos com DM não eram alfabetizados, o que dificultava o cumprimento das prescrições médicas sem auxílio. Isto limita a autonomia do idoso e exige maior cuidado na prescrição e orientação profissional ${ }^{(24)}$, e embora os idosos fossem detentores de algum grau de conhecimento referente ao DM e aos seus cuidados, seguiam apenas parcialmente as orientações dos profissionais ${ }^{(26)}$.

A restrição alimentar destacou-se como outro fator que acarreta a não adesão à dieta, consistindo no ponto de maior repercussão sobre o modo de viver (E7). Na opinião dos idosos, poder ingerir aquilo que se deseja, de acordo com sua cultura alimentar e especificidades individuais, é fundamental para se sentirem com autonomia e qualidade de vida ${ }^{(16)}$. Assim, a dieta alimentar torna-se um obstáculo ${ }^{(16)}$. O uso do álcool pelos idosos, (E12) também mostrou-se como dificultador da dieta alimentar, e apenas $3,3 \%$ realizavam "regime para perder peso" ${ }^{(25)}$.

Neste cenário, percebe-se a importância do apoio profissional e dos grupos de convivência nos estabelecimentos de saúde, que, por meio do diálogo e de orientações, compreendendo o ser na sua essência, podem auxiliar na modificação dos hábitos de vida. Salienta-se que as pessoas com diabetes não compreendem os reais malefícios da não adesão à terapêutica e do suporte derivado do auxílio profissional $(\mathrm{E} 7)^{(16)}$.

Quanto à percepção de saúde, foi evidenciado (E6, E8) que os idosos a consideravam como ruim/ muito ruim, má ou péssima ${ }^{(15,17)}$. As suas visões de mundo eram repletas de limitações e de sentimentos de ameaças à saúde, pois o seu viver estava atrelado às condições restritas de alimentação e de estilo de vida, revelando a sua condição de diabético como algo ruim, em um enfrentamento contínuo da morte, vivendo sob um universo de ameaça à vida e não em busca de qualidade de vida. A determinação de como irão conduzir suas práticas de cuidado diário depende de como entendem suas realidades $(\mathrm{E} 10)^{(19)}$.

A complicação mais frequente encontrada (E5, E11) foi a diminuição da acuidade visual pela retinopatia diabética ${ }^{(14,20)}$. O surgimento de novas complicações e/ou doenças necessita introdução de novos medicamentos, podendo acarretar em interações medicamentosas e mais efeitos colaterais.

Diante do exposto, verifica-se a existência de lacunas na educação, promoção e prevenção dos agravos pelos profissionais de saúde (E9). Estes dados demonstram a preocupação referente à evolução da doença, podendo comprometer a autonomia e a independência dos idosos para as atividades de vida diária e atividades instrumentais de vida diária. É importante que os idosos com DM estejam cientes de sua condição de saúde e sejam informados sobre a seriedade do regime terapêutico ${ }^{(18)}$.

Para isso, os profissionais de saúde carecem de trabalho efetivo e focado no desenvolvimento de abordagem educativa sobre os malefícios que a DM provoca na pessoa idosa. É preciso diálogo efetivo, com idosos e familiares, para esclarecer e prestar informações relevantes que garantam a segurança do indivíduo com DM, e sua conscientização acerca da importância da adesão à terapêutica.

\section{CONSIDERAÇÕES FINAIS}

Este estudo permitiu a identificação na literatura dos fatores que influenciam a adesão do idoso com Diabetes Mellitus ao tratamento recomendado. A pesquisa baseada em evidências, por meio da revisão integrativa, com dados extraídos e organizados e as informações ordenadas e analisadas por meio da análise textual, mostrou-se adequada para a formação de categorias e alcance dos objetivos propostos. 
Como facilitador da adesão à terapêutica, encontrou-se o apoio familiar, apresentado como suporte psicológico, principalmente na adaptação ao plano alimentar. O apoio familiar, dos grupos de convivência e dos profissionais de saúde mostram-se fundamentais para aderência ao tratamento e a manutenção do controle glicêmico, além da própria religiosidade do idoso.

Como fatores que influenciam negativamente a adesão ao tratamento, verificaram-se a presença de complicações e de outras comorbidades, os efeitos colaterais dos medicamentos e a sua não disponibilização contínua e segura pelas Unidades Básicas de Saúde, dificultando assim a efetivação correta, a baixa renda dos idosos e baixo nível de escolaridade.

Entende-se a necessidade da intensificação da atenção aos idosos com Diabetes Mellitus no Brasil. Os enfermeiros, principalmente, necessitam despertar para atuações de coordenação, assistência, educação, pesquisa sobre esta temática. Isto implicará no aumento de ações educativas, assim como de pesquisas que, além da obtenção de informações sobre os hábitos de vida, enfoquem também as melhores formas de abordagem dessa parcela da população, considerando seus fatores de risco. No intuito do estabelecimento de um diálogo conscientizador, compatível com suas demandas e necessidades, preserva-se a manutenção da qualidade de vida, autonomia e independência, e melhor elaboração de ações preventivas.

\section{REFERÊNCIAS}

1. International Diabetes Federation (IDF). IDF Diabetes Atlas. $7^{a}$ ed. [Internet] Brussels: International Diabetes Federation; 2015 [acesso em 08 jun 2015]. Disponível: http://www.diabetesatlas.org/.

2. Ministério da Saúde (BR). Secretaria de Atenção à Saúde. Departamento de Atenção Básica. Estratégias para o cuidado da pessoa com doença crônica: Diabetes Mellitus. [Internet] Brasília: Ministério da Saúde; 2013 [acesso em 04 abr 2015]. Disponível: http://189.28.128.100/dab/docs/portaldab/publicacoes/caderno_36.pdf.

3. Ministério da Saúde (BR). Secretaria de Atenção à Saúde. Departamento de Atenção Básica. Envelhecimento e saúde da pessoa idosa. Cadernos de Atenção Básica № 19. Brasília: Ministério da Saúde; 2006.

4. Minayo MCS, Hartz ZMA, Buss PM. Qualidade de vida e saúde: um debate necessário. Ciênc. saúde coletiva. [Internet] 2000; 5(1) [acesso em 13 jun 2015]. Disponível: http://dx.doi.org/10.1590/S1413-81232000000100002.

5. Mendes KDS, Silveira RCCP, Galvão CM. Revisão Integrativa: Método de pesquisa para a incorporação de evidencias na saúde e na enfermagem. Texto Contexto Enferm. [Internet] 2008;17(4) [acesso em 13 jun 2015]. Disponível: http://dx.doi.org/10.1590/S0104-07072008000400018.

6. Stone PW. Popping the (PICO) question in research and evidence-based practice. Appl Nurs Res. [Internet] 2002;15(3) [acesso em 22 out 2016]. Disponível: http://dx.doi.org/10.1053/apnr.2002.34181.

7. Brasil. Lei n. 10.741, de 1 de outubro de 2003. Dispõe sobre o estatuto do idoso e dá outras providências. Diário Oficial da União [Internet] 3 out 2003 [acesso em 13 fev 2016]. Disponível: http://www.planalto.gov.br/ccivil_03/ leis/2003/L10.741.htm.

8. Melnyk BM, Fineout-Overholt E. Making the case for evidence-based practice. In: Melnyk BM, FineoutOverholt E. Evidence based practice in nursing \& healthcare. A guide to best practice. Philadelphia: Lippincot Williams \& Wilkins;2005.p.3-24.

9. Moraes R. Análise textual qualitativa entendida como processo integrado de aprender, comunicar e interferir em discursos. In: Galiazzi MC, Freitas JV, organizadores. Metodologias emergentes de pesquisa em educação ambiental. Ijuí: Unijuí; 2005. p.85-114.

10. Tavares DMS, Rodrigues RAP. Educação conscientizadora do idoso diabético: uma proposta de intervenção do enfermeiro. Rev. esc. enferm. USP. [Internet] 2002;36(1) [acesso em 14 jun 3015]. Disponível: http://dx.doi. org/10.1590/S0080-62342002000100013.

11. Ruiz T, Chalita LVAS, Barros MBA. Estudo de sobrevivência de uma coorte de pessoas de 60 anos e mais no município de Botucatu (SP) - Brasil. Rev. bras. epidemiol. [Internet] 2003;6(3) [acesso em 25 de out 2016] Disponível: http://dx.doi.org/10.1590/S1415-790X2003000300006. 
12. Vasconcelos FF, Victor JF, Moreira TMM, de Araújo TL. Utilização medicamentosa por idosos de uma Unidade Básica de Saúde da Família de Fortaleza - CE.Acta paul. enferm. [Internet] 2005;18(2) [acesso em 13 jun 2015]. Disponível: http://dx.doi.org/10.1590/S0103-21002005000200010.

13. Viegas-Pereira APF, Rodrigues RN, Machado CJ. Fatores associados à prevalência de diabetes auto referido entre idosos de Minas Gerais. Rev. bras. estud. popul. [Internet] 2008;25(2) [acesso em 24 out 2016]. Disponível: http://dx.doi.org/10.1590/S0102-30982008000200011.

14. Cordeiro RC, Jardim JR, Perracini MR, Ramos LR. Fatores associados ao equilíbrio funcional e à mobilidade em idosos diabéticos ambulatoriais. Arq Bras Endocrinol Metab. [Internet] 2009;53(7) [acesso em 24 out 2016]. Disponível: http://dx.doi.org/10.1590/S0004-27302009000700007.

15. Francisco PMSB, Belon AP, Barros MBA, Carandina L, Alves MCGP, Goldbaum M, et al. Diabetes auto-referido em idosos: prevalência, fatores associados e práticas de controle.Cad. Saúde Pública.[Internet] 2010;26(1) [acesso em 13 jun 2015]. Disponível: http://dx.doi.org/10.1590/S0102-311X2010000100018.

16. Ribeiro JP, Rocha AS, Popim RC. Compreendendo o significado de qualidade de vida segundo idosos portadores de diabetes mellitus tipo II. Esc. Anna Nery. [Internet] 2010;14(4) [acesso em 14 jun 2015]. Disponível: http://dx.doi.org/10.1590/S1414-81452010000400016.

17. Mendes TAB, Goldbaum M, Segri NJ, Barros MBA,Cesar CLG, AlvesMCGP.Diabetes mellitus: fatores associados à prevalência em idosos, medidas e práticas de controle e uso dos serviços de saúde em São Paulo, Brasil. Cad. Saúde Pública. [Internet] 2011;27(6) [acesso em 13 jun 2015]. Disponível: http://dx.doi.org/10.1590/ S0102-311X2011000600020.

18. Gandra FPP, Silva KC, Castro CF, Esteves EA, Nobre LN. Efeito de um programa de educação no nível de conhecimento e nas atitudes sobre o Diabetes Mellitus. Rev Bras Promoç Saúde. [Internet] 2011;24(4) [acesso em 24 out 2016]. Disponível: http://dx.doi.org/10.5020/2089.

19. Souza NMG, Honorato SMA, Xavier ATF, Pereira FGF, de Ataíde MBC. Visão do mundo, cuidado cultural e conceito ambiental: o cuidado do idoso com Diabetes Mellitus. Rev. Gaúcha Enferm. [Internet] 2012;33(1) [acesso em 14 jun 2015]. Disponível: http://dx.doi.org/10.1590/S1983-14472012000100019.

20. Marques MB, da Silva MJ, Coutinho JFV, Lopes MVO. Avaliação da competência de idosos diabéticos para o autocuidado. Rev.esc.enferm.USP. [Internet] 2013;47(2) [acesso em 14 jun 2015]. Disponível: http://dx.doi. org/10.1590/S0080-62342013000200020.

21. de Sousa MC, Dias FA, Nascimento JS, Tavares DMS. Correlation of quality of life with the knowledge and attitude of diabetic elderly. Invest. educ. enferm. [Internet] 2016;34(1) [acesso em 24 out 2016]. Disponível: http:// dx.doi.org/10.17533/udea.iee.v34n1a20.

22. Salbego LP, da Silveira A, Ramos AK, Pez APZ, Hammerschmid KSA. Inserção da família no gerenciamento do cuidado ao idoso com Diabetes Mellitus. Rev enferm UFPE on line. [Internet] 2013;7(12) [acesso em 15 jun 2015]. Disponível: http://www.revista.ufpe.br/revistaenfermagem/index.php/revista/article/view/4935/pdf_4152.

23. Araújo KO, Andrade AN, Costa TS, de Freitas MA, do Nascimento MMP, da Silva EN. Qualidade de vida de portadores de Diabetes Mellitus Tipo 2. Rev enferm UFPE on line. [Internet] 2013;7(9) [acesso em 14 jun 2015]. Disponível: http://www.revista.ufpe.br/revistaenfermagem/index.php/revista/article/view/4057/pdf_3416.

24. Tavares DMS, Drumond FR, Pereira GA. Condições de saúde de idosos com diabetes no município de Uberaba, Minas Gerais. Texto Contexto Enferm .[Internet] 2008;17(2) [acesso em 22 mai 2015]. Disponível: http:// dx.doi.org/10.1590/S0104-07072008000200017.

25. Castro ARV, Grossi SAA. Custo do tratamento do diabetes mellitus tipo 1: dificuldades das famílias. Acta paul. enferm.[Internet] 2008;21(4) [acesso em 14 jun 2015]. Disponível: http://dx.doi.org/10.1590/S010321002008000400014.

26. Lenardt MH, Hammerschmidt KSA, Borghi ACS, Vaccari E, Seima MD. O idoso portador de nefropatia diabética e o cuidado de si. Texto Contexto Enferm. [Internet] 2008;17(2) [acesso em 06 mai 2015]. Disponível: http://dx.doi.org/10.1590/S0104-07072008000200013. 